\title{
Disease Information Through Comics: A Graphic Option for Health Education
}

\author{
Josh Rakower ${ }^{1} \cdot$ Ann Hallyburton ${ }^{1}$
}

Accepted: 9 December 2021 /Published online: 17 January 2022

(c) The Author(s), under exclusive licence to Springer Science+Business Media, LLC, part of Springer Nature 2022

\begin{abstract}
This paper presents a critical interpretive synthesis of research on the efficacy of comics in educating consumers on communicable diseases. Using this review methodology, the authors drew from empirical as well as non-empirical literature to develop a theoretical framework exploring the implications of comics' combination of images and text to communicate this health promoting information. The authors examined selected works' alignment with the four motivational components of Keller's ARCS Model (Attention, Relevance, Confidence, and Satisfaction) to evaluate research within the context of learner motivation. Findings of this research indicate comics offer a useful method for providing consumer health education, particularly regarding topics that individuals may have difficulty in discussing openly.
\end{abstract}

KEYWORDS Comic books, strips, etc. · Graphic novels $\cdot$ Communicable diseases $\cdot$ Health education

\section{INTRODUCTION}

Health messaging, particularly regarding communicable disease, spreads through a variety of media. Whether beneficial or harmful, effective or barely noticeable, transmission can be as potentially virulent as an uncovered cough or an overused metaphor. What makes such messaging take hold includes everything from the relatability of new mother-focused anti-vaccine memes and photos shared via Facebook (Bradshaw et al. 2021), to emotional imagery and sound in a YouTube video (Phan et al. 2021), to a humorous, animal-themed poster on the door of a clinic. While purely text based messages are commonplace for relaying health information, a mixture of text and graphical images may be more effective (Niederdeppe et al. 2019). Information presented strictly as text can be overwhelming, leaving readers with a sense of information overload (Lee, Lee-Geiller, and Lee 2020). With their mixture of images, limited text, and comparatively short format, comics provide an option for effectively communicating health messages (Branscum and Sharma 2009). Despite spawning an ever-growing graphic

Josh Rakower

jrakower@wcu.edu

1 Western Carolina University, Hunter Library, Cullowhee, NC, USA 
medicine movement (Saji, Venkatesan, and Callender 2021), the format's usage in healthcare is limited, leaving consumers unaware such accessible media exist (McNicol 2017).

Critically acclaimed comics such as Blue Pills: A Love Story by Frederik Peeters (2008) and Monsters by Ken Dahl (2009) individually tell stories about the health, emotional, and social impacts of communicable diseases (Williams 2012a; 2012b; Olson 2009). Do such comics integrate useful information, increase awareness, or influence action in their consumers, though? Individual studies have yielded mixed results regarding impacts of health messages presented in comic form (Albright and Gavigan 2014; Sheer et al. 2018). The current review offers a synthesizing argument on the efficacy of comics in sharing communicable disease information by looking across multiple studies and publications using critical interpretive synthesis (CIS) methodology (Dixon-Woods et al. 2006). To explore mechanisms of how comics might work as health information and education tools, individual publications are evaluated using a model of human motivation and behavior (Fig. 1).
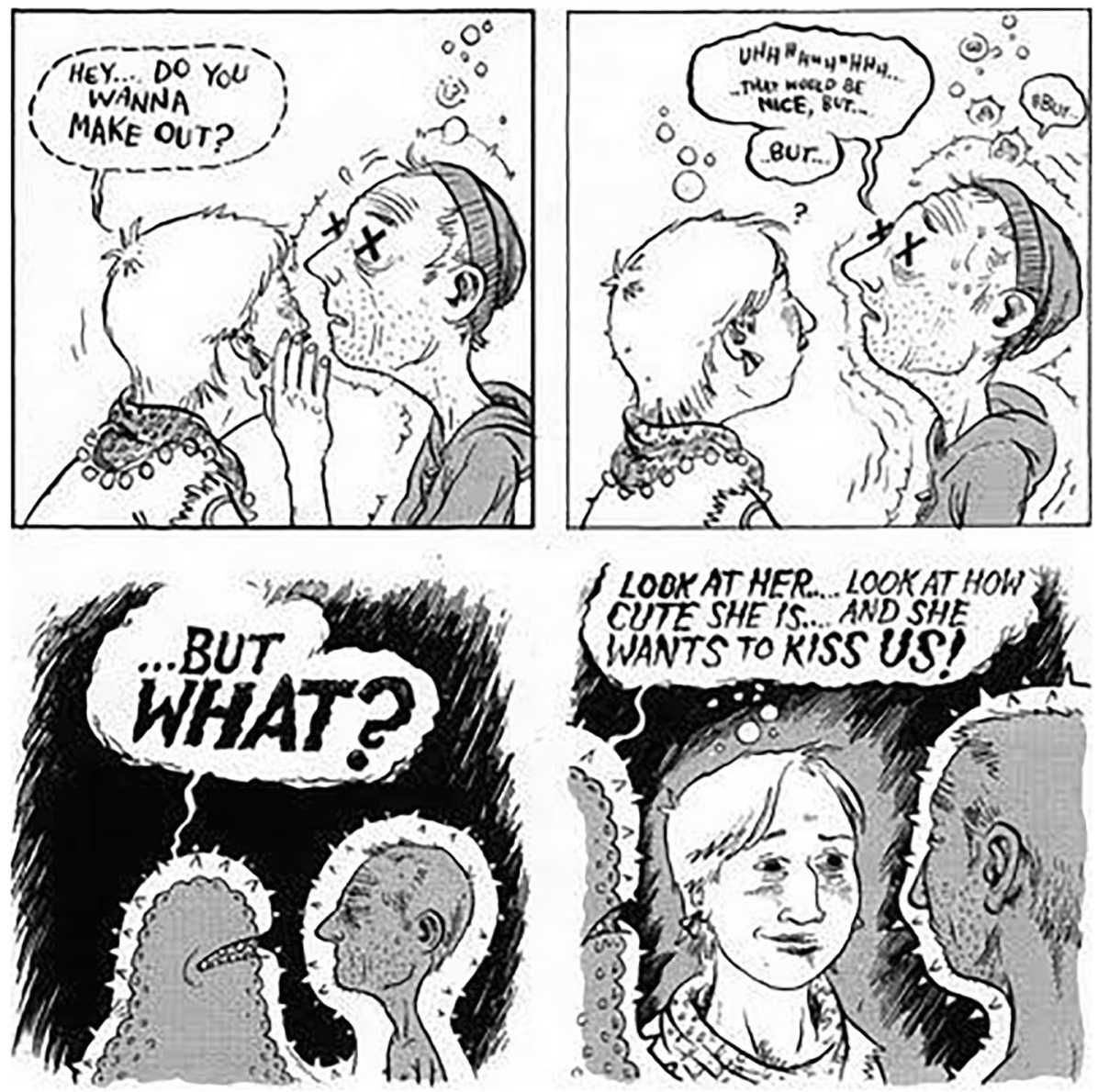

Fig. 1 Panels from Dahl's Monsters depicting the indecision of a character with Herpes Simplex 1 in a romantic situation (Dahl 2009, 83) 


\section{METHODS}

\section{Defining "comics"}

Exploring the issue, setting inclusion and exclusion criteria, material selection, and synthesis itself required the authors to adopt a fixed definition of "comics." As comic theorist Scott McCloud discusses in his classic book Understanding Comics: The Invisible Art (1993), though most people recognize comics when they encounter them, defining the medium is a much more difficult task. McCloud characterizes comics as "Juxtaposed pictorial and other images in deliberate sequence, intended to convey information and/or to produce an aesthetic response in the viewer" $(1993,20)$. McCloud admits this explanation is imperfect; the definition includes works such as comic books and strips and graphic novels but excludes single panel cartoons such as The Far Side. The term "pictorial image" is not limited to illustrations and allows photocomics (fotonovelas) employing photographs with text to meet the definition of comic. McCloud reiterates this description in his 2006 book Making Comics, stating that the stories comics tell "need to take the form of images in sequence, perhaps with words" (9). McCloud maintains this definition is not a permanent description, though. As McCloud notes, new generations will redefine and reimagine comics $(1993,23)$.

\section{Formulating the review question}

The role comics play in providing health-related information seemed particularly relevant to the authors who are academic librarians specializing in health information or with an interest in comics as a medium. The authors primarily serve undergraduate and graduate students who need health information as consumers or as healthcare providers and educators. As librarians, they handle questions as to whether comics and similar works are appropriate, not only for recreational reading and literary collections, but for the health sciences collections and educational resources as well.

This CIS methodology guiding this review integrates an array of publication types and encourages re-evaluation throughout the process, culminating in construction of a new conceptual model of the topic. Pioneered within healthcare research, authors have since employed CIS within education (Markoulakis and Kirsh 2013; Zhang, Basham, and Yang 2020), library science (Bales and Gee 2013; Bawden 2012), linguistics (Masson et al. 2021), musicology (McFerran 2016; West and Silverman 2021), and social sciences (Depraetere et al. 2020; Poirier, Grépin, and Grignon 2020) research. The CIS approach Dixon-Woods and colleagues created emerged from their belief that other synthesis methodologies of the time "fail to be sufficiently critical, in the sense of offering a critique. There is rarely an attempt to reconceptualise the phenomenon of interest, to provide a more sweeping critique of the ways in which the literature in the area have chosen to represent it, or to question the epistemological and normative assumptions of the literature" (DixonWoods et al. 2006, 43). The CIS process critically considers together a wide body literature encompassing qualitative and quantitative research and other literature types with relevance to the topic under consideration. This framework seemed appropriate for a review of the health education efficacy of a format that mixes visual art and literature.

Aligning to CIS methods, question refinement was iterative with initial queries focusing on studies of sexual health messages in comics. Early literature searches yielded relevant 
research; however, formation of a cohesive, relevant question was elusive. The COVID-19 pandemic, combined with a surrounding infodemic of misinformation, prejudice, and early research extrapolated to widely applicable fact (Mheidly and Fares 2020) influenced the authors to broaden their scope to all types of communicable disease, including COVID-19. Research on STIs identified earlier would remain relevant while illnesses spread through means other than intimate contact would be included.

\section{Theoretical framework}

The authors looked for an established model of human behavior to use in evaluating texts to provide a more accurate exploration of how comics might influence individuals' health behaviors. Originally the authors planned to apply as a theoretical framework Ajzen's Theory of Planned Behavior (TPB), a popular model used in public health and other arenas to predict and explain human behaviors. TPB posits that intention and ability help determine action (Ajzen 1991; 2002). TPB seemed suitable as a framework for examining how exposure to comics dealing with communicable disease might impact readers' behaviors. Though the theory is widely used and initially seemed appropriate, applying the theory's concepts of behavioral intent, attitude, societal subjective norms, and perceived personal control over behaviors is surprisingly difficult. As the authors progressed in analyzing selected articles, they found aligning TPB concepts incongruous within the specific context of the study.

Instead, the authors were drawn to Keller's (1987) ARCS Model of Motivation. The ARCS Model of Motivation is based around four constructs: Attention, Relevance, Confidence, and Satisfaction. Although the ARCS Model is more prominently used in the field of education, it has previously received limited application within healthcare (Al-Tawfiq and Pittet 2013; Stockdale, Sinclair, and Kernohan 2014).

As the authors independently analyzed selected articles, they both found application of the ARCS Model of Motivation a more natural fit than TPB. Although TPB has been shown to be an effective model for evaluating the messaging of health promotional materials, the authors were less certain about its ability to be used in evaluating mediums and methods of delivering a message. As the synthesis focuses on comics as a tool in communicating health information, this application of the model offered a more workable framework.

Evaluation of publications includes the extent to which comics engage attention, relevance of messaging, whether viewing comics imparts information in a way to imbue confidence in understanding the information, and the overall feeling of satisfaction comic consumers feel after viewing the material. In the case of empirical research, study participants fulfill the role of comic consumers. For publications not covering empirical research (e.g., content analyses, literary criticism, and theoretical articles), the consumer is assumed to be the population discussed or, in some cases, the article's author(s).

\section{Searching the literature}

Searches for relevant literature were conducted, expanded, revised, and repeated throughout 2020 and early 2021. In accordance with CIS methodology, the authors followed an "organic process that fitted better with the emergent and exploratory nature of the review question[s]" (Dixon-Woods et al. 2006, 3) than the highly regimented and, for this purpose, overly limited searching process common in other systematically conducted reviews. 
Systematic search methodologies were interwoven, though, and an example search is included to enable a measure of reproducibility for future researchers.

Resources were searched using terms related to comics, graphic novels, and common human-to-human communicable diseases and issues textually described generally as communicable diseases. Resource-specific subject headings were mined for additional applicable terms. An example of one search phrase is:

(comic OR comics OR "graphic novel*" OR "graphic medic*" OR "graphic narrative*" OR manga) AND ("communicable disease*" OR infect* OR HIV OR syphilis OR gonorrhea OR virus* OR "sexual*transmi*” OR influenza* OR germ OR germs OR coronavirus OR vaccine* OR immuniz* OR immunis* OR "public health")

This phrase, variations of it, and simpler keywords were used to search healthcare, information science, education, social sciences, and general resources. Those resources included the databases Cumulative Index to Nursing and Allied Health Literature (CINAHL), Education Source, ERIC, Library, Information Science \& Technology Abstracts (LISTA), MEDLINE, SocINDEX, and PsycINFO, as well as multidisciplinary databases such as Academic Search. Multiple searches of both Google Scholar and the general Google search engine were conducted. Article and book references were also explored.

Both authors undertook independent and often replicative searches. The authors recognize some relevant materials were likely not discovered through the authors' methods of searching or were missed due to misinterpretations of publications' adherence to inclusion criteria. As the authors' primary reading language is English, only results published in English were considered. While searches were thorough, they were not exhaustive. Extensive summation of available data is not desired in CIS as "the focus in interpretive synthesis is on the development of concepts and theory rather than on exhaustive summary of all data" (Dixon-Woods et al. 2006, 3). Publication date limitations were not applied due to the topic's timeless nature, though selected publications all emerged since wide acknowledgement of the global HIV/AIDS pandemic in the mid-1980's (Clark and Coppola 1985; Richards 1985). While HIV/AIDS is quite far from the first communicable disease, its emergence roughly coincided with recognition of the formal scholarship of comics (Coughlin 1990).

\section{Sampling}

This synthesis applies specific exclusion and inclusion criteria aimed at limiting selection bias on the part of the authors while also enabling a manageable sample size of publications. The authors initially planned to include only quantitative and qualitative studies using comics as a health information intervention. Though CIS methodology embraces use of multiple types of research, Dixon-Woods' and colleagues' (2006) original CIS example focuses more on usage of multiple forms of empirical research. As the authors of the current synthesis learned more about their topic and encountered fewer such studies than desired, the authors agreed to also include theoretical works, content analyses, literary criticism and critical reflections, and other information syntheses when relevant. Support for this expanded criteria can be found in the CIS work of McKibbin, Humphreys, and Hamilton which includes not just "qualitative and quantitative empirical studies," but also "systematic reviews, conceptual papers and reports on policy" $(2016,659)$. Further, Flemming and McInnes also affirm CIS "explicitly allows the integration of qualitative and quantitative research and also theoretical papers" $(2012,61)$. 
In searching with the example phrase, approximately five hundred eighty publications were reviewed at least at the title level by both authors. From that grouping, as well as relevant materials identified during earlier iterations of the search process, forty journal articles and one dissertation were selected for review in their entirety. Of that number, twentythree journal articles and one dissertation were determined to meet inclusion criteria for the analysis.

\section{Data extraction}

The authors independently extracted and recorded data from the twenty-four publications. Factors drawn from each publication include: title, article author(s), publication date, comic format, study population and size (for empirical research), outcomes or conclusions, and, critically, applicability of the four constructs of the ARCS Model of Motivation (Attention, Relevance, Confidence, and Satisfaction). Author disagreements in coding were noted and discussed. Where consensus could not be reached, or when authors concurred they could not determine whether ARCS Model constructs were in evidence, elements were noted as "not evident." In cases where construct application was not feasible (e.g., publication provides analysis of a comic rather than comics' effect on readers), constructs were labeled "not applicable." Table 1 offers a simplified version of the authors' combined coding matrix. Notations of whether constructs exist come from the authors' subjective understanding of both the analyzed publications and the ARCS Model.

\section{RESULTS}

\section{ARCS Model constructs as themes}

\section{Attention}

In the context of the ARCS Framework, Keller calls attention a "prerequisite for learning" and emphasizes that, to successfully motivate learners, attention must be both captured and sustained $(1987,3)$. The authors reached consensus that seventeen of the twenty-four works clearly included themes related to attention. Most publications noted the use of personal narratives, humor, and other forms of entertainment to get and maintain readers' attention.

Five of the studies (Aguolu 2018; Bakker 1999; Beck 2006; Murray-Román 2019; Scavone et al. 2019) discussed the use of humor in comics as a means of stimulating the attention of readers. Much like superheroes, humor is a common theme in the medium of comics. Although humor is not unique to comics, the mixture of pictorial images and text allow creators to portray humor in unique ways.

In her dissertation, Aguolu (2018) also explores the efficacy of using a comic to promote Human Papillomavirus Virus (HPV) vaccination within a university population. Citing low HPV vaccine uptake among students in higher education, Aguolo, with advisors Phillips and Smith, created a student-focused, theory-based HPV vaccine comic they posit might have greater effectiveness than more traditional educational interventions due to comics' "availability, portability, attention-grabbing, use of simple language, and visual cues" $(2018,77)$.

Aguolu's (2018) dissertation, as well as the works of de Castro Verla Cruz and colleagues (2019), and Obare and colleagues (2013) discuss the use of color in comics to help 


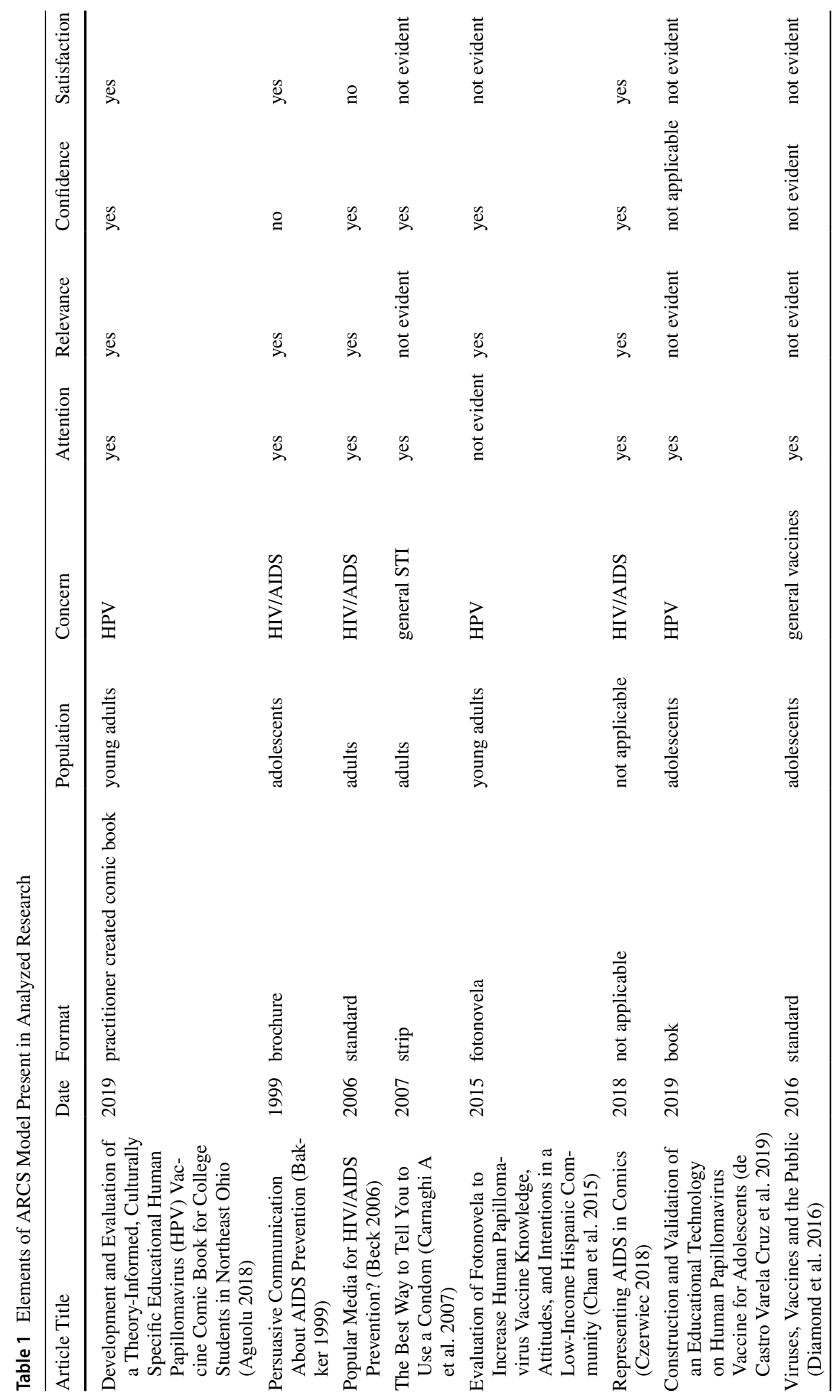




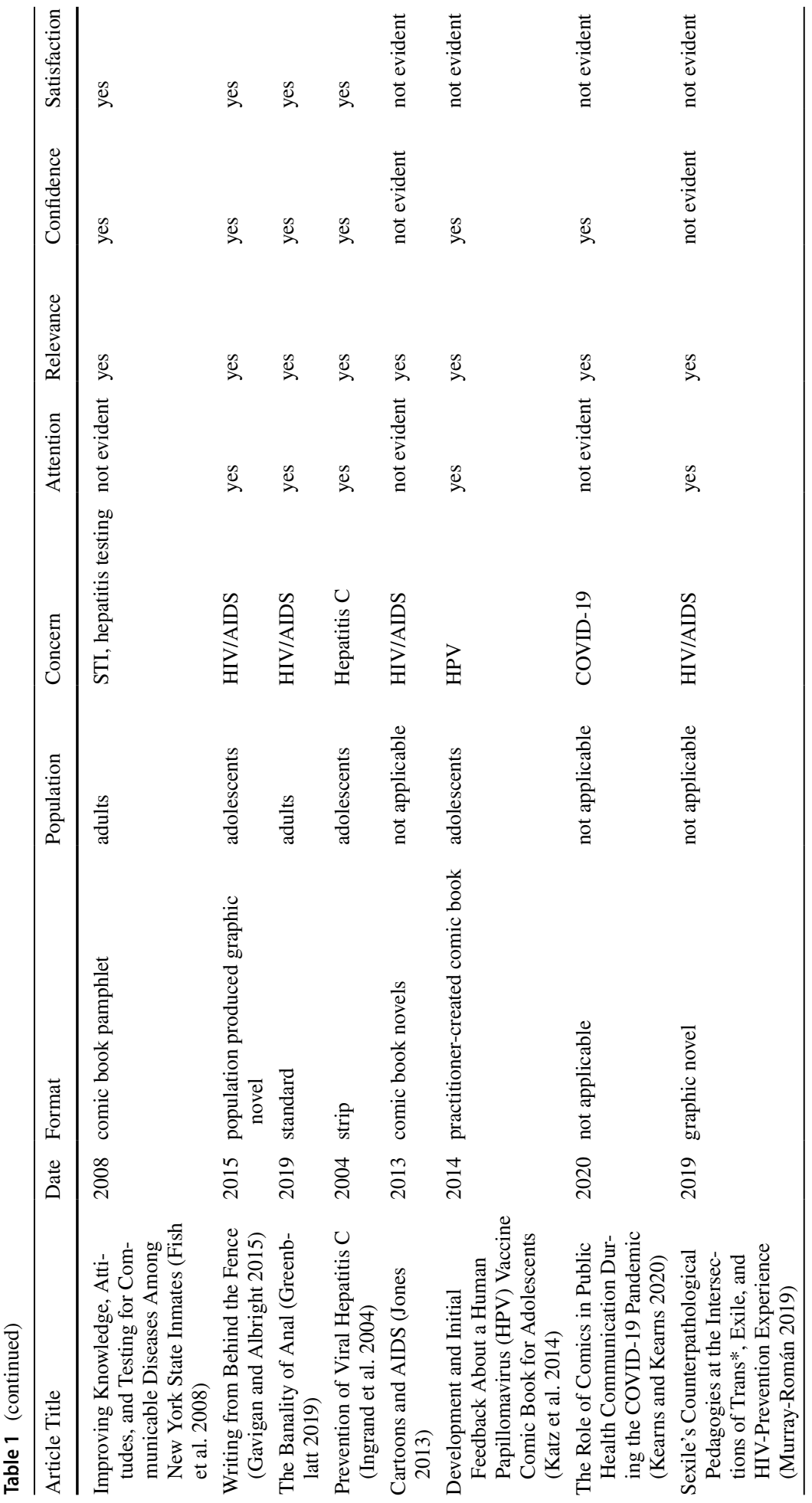




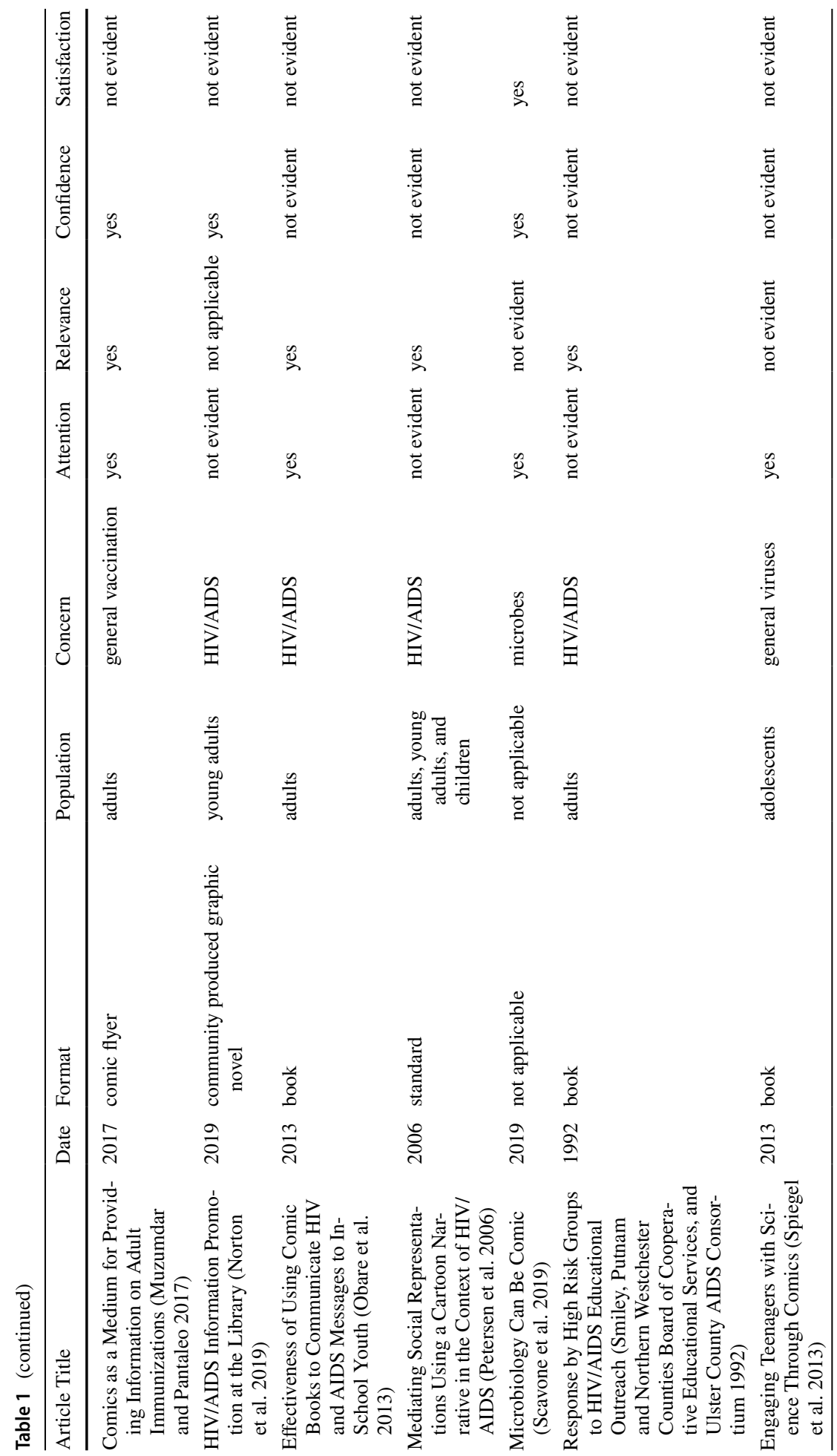




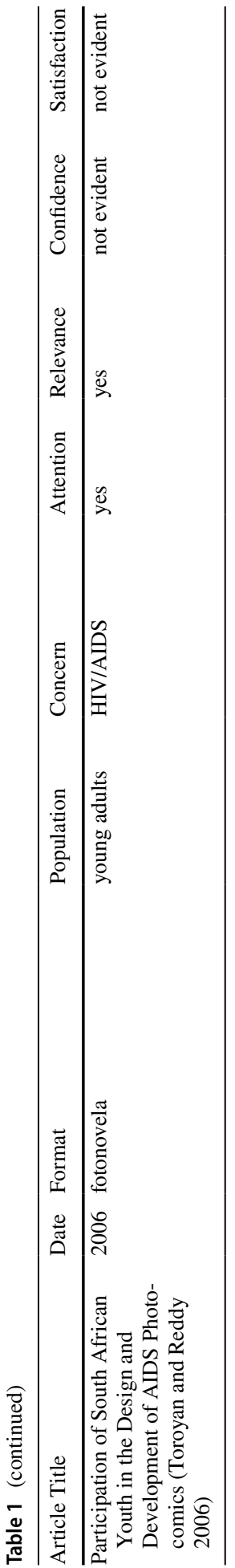


grab the attention of younger audiences. These three studies employed color when designing their original comics to draw their audiences' attention. In their study, de Castro Verla Cruz and colleagues (2019) found that their target audience of adolescents responded positively to the combined storytelling and colorful drawings designed to inform them about HPV vaccination.

\section{Relevance}

Keller refers to relevance as "people's feelings or perceptions of attraction toward desired outcomes, ideas, or other people based upon their own goals, motivations, and values" (2010, 98). When coding for relevance as a theme, the authors sought examples where content was designed to appeal to a specific audience. The authors found consensus on the presence of such an interpretation of relevance in eighteen of the twenty-four works. The most common examples of relevance are shown in studies using comics with characters tailored to their intended audiences. For example, a 2006 study by Beck focused on Swahili HIV/AIDS prevention comics published in the magazine Kingo. These comics were drawn by local artists and featured characters and locations familiar to local consumers. Beck (2006) maintains that comics used for teaching must be in harmony with local realities, or else the comics' messages will be rejected.

Comics creator and theorist McCloud posits that readers may be more able to identify with cartoon-style characters than with photos of real people or photo-realistic characters. By removing details that make characters unique, cartoon imagery renders characters more universal and thus more easily relatable (McCloud 1993). Both Muzumdar and Pantaleo (2017) in their study of a CDC-produced comics' effects on attitudes toward immunization in adults and Petersen and colleagues' (2006) study of comics' efficacy to promote health behavior change related to HIV/AIDS in South African families cite this concept of illustrations' relatability as a reason why comics can be an effective tool for providing health information. However, Toroyan and Reddy (2006) found in their research with South African youth that photocomics or fotonovelas, comics using photographs instead of cartoon imagery, were effective due to the photos' grounding of the comics in the real world. Similarly, a study of young adults in Southern California found a fotonovela featuring photos of Latinx characters made those characters' interactions with HPV vaccines more relatable (Chan et al. 2015). These disparate findings suggest that a range of graphical styles can be useful in increasing relevance for a target audience.

\section{Confidence}

ARCS Model creator Keller refers to confidence as "people's expectancies for success in the various parts of their lives" $(2010,135)$. When coding for examples of the thematic construct of confidence, the authors looked for instances where the comics medium is used in a way that either helps readers increase their belief that they can achieve desired healthrelated outcomes or strengthen their confidence in their ability to understand the content being presented. Overall, the authors reached consensus that fourteen of the twenty-four articles included themes related to confidence.

In her research on HIV/AIDS-related messaging within two popular comics in Swahilispeaking populations, Beck (2006) finds comics to be an effective method of spreading public health messages to individuals who have low or no literacy as well as to individuals who are literate. Likewise, Carnaghi and colleagues report finding the comic format 
efficacious in communicating sexual health messages, with a caveat. Basing their study around the Theory of Reasoned Action, the researchers hypothesized that Italian high school students receiving an intervention of a comic or a pamphlet would have greater knowledge of the topic than those that had not received such information (the control group). Study results supported this hypothesis. However, after assessing study participants using the Need for Cognition Scale, the authors found that participants evincing a lower desire for cognitive stimulation benefited more from the comic strip intervention, while participants scoring higher on the Need for Cognition scale showed lesser benefit from the comic intervention. In that study, the more text-heavy pamphlet produced better results with students desiring higher levels of cognitive stimulation (Carnaghi A et al. 2007).

\section{Satisfaction}

Within the context of the ARCS Model, satisfaction is understood as reinforcing accomplishments with either internal or external rewards (Keller 2010). Since comics are commonly used for entertainment, the authors hypothesized that this thematic concept would be present more often than other ARCS constructs. Overall, the authors agreed on the involvement of the theme of satisfaction in just eight of twenty-four works.

When satisfaction is viewed in the context of comic consumers' expression of enjoyment, relatively few publications discuss the concept (Aguolu 2018; Bakker 1999; Scavone et al. 2019). While an article by Fish and colleagues also addresses the enjoyment provided by comics, the study it details employs multiple media types as an intervention. Fish and colleagues' study used an educational video as well as a comic book-style pamphlet as they sought to increase knowledge of and motivation toward obtaining testing for STIs in adults incarcerated in correctional facilities in the state of New York (Fish et al. 2008).

In addition to enjoyment, satisfaction may also be viewed in the context of a feeling of relief or achievement. Although most studies analyzed comics as a medium to be consumed, several studies investigated the creative process behind comics dealing with communicable disease. M. K. Czerwiec, a nurse and comic creator, wrote about her experience creating comics dealing with her time working with individuals with AIDS. Czerwiec discusses her process for creating comics and how this procedure served as a means of catharsis for her (Czerwiec 2018). Similarly, Gavigan and Albright detail their work with young creators in a juvenile detention facility. The students in the facility collaborated with an illustrator to create a teen-targeted graphic novel dealing with HIV/AIDS. The researchers found that the youth who helped create the educational comic felt a strong sense of satisfaction which remained with them for years (Gavigan and Albright 2015).

\section{DISCUSSION}

\section{Limitations}

In analyzing publications where ARCS Model constructs are not specifically noted, the authors of the current research made inferences from study and research findings. They noted and discussed how those findings were more broadly applicable to Attention, Relevance, Confidence, and Satisfaction. Aside from this need for inference, the authors themselves had trouble in reaching consensus between their individual interpretations of ARCS Model constructs, particularly in regard to Satisfaction. 
Within the ARCS Model, the construct of Satisfaction is considered to be a reinforcing accomplishment yielding some kind of reward (Keller 2010). Satisfaction may be internal, such as a sense of achievement or enjoyment. Satisfaction may also come through external means; for instance, the creation of a tangible artifact like a comic book may provide satisfaction. As previously noted, the comic medium is strongly associated with entertainment. Thus, a broad interpretation of the construct of Satisfaction may hold that readers of comics inherently feel some sense of enjoyment or achievement, even if not specifically discussed or measured. One author held this interpretation and noted a majority of reviewed publications as including satisfaction as a theme. The other author took a more conservative approach, requiring tangible indications that satisfaction was addressed or measured within publications. This discrepancy might suggest that this particular construct of the ARCS Model poses greater difficulty than others when dealing with a topic commonly associated with entertainment. However, the disconnect may not seem so great in future analyses that do not employ CIS. CIS by nature encourages continual critique and evolving theoretical development, a recipe for divergent interpretations where individual conclusions are not right or wrong as long as they are supported.

The difficulty possibly created by using the ARCS Model and CIS methodology in tandem for analysis involving an entertainment-related medium was unforeseen. However, that difficulty should likely not be viewed as a prohibition against using the tools together in the future. While the ARCS Model enabled a deeper analysis of the motivational mechanisms at work in comic interventions, the CIS methodology encouraged reflexive response to what the authors learned by considering the reviewed materials and the research question through this lens.

The CIS methodology allowed the authors to modify their review question as world events (notably the COVID-19 pandemic) and a limited amount of published research pertaining to their original query called for a change in focus. The review question transitioned from examining efficacy of sexual health information in comics to analysis of educational outcomes from consumption of comics featuring communicable disease information.

Further, the method enabled search, selection, and analysis mechanisms to evolve through much of the synthesizing process. Not only did the change in the research question necessitate alteration of search terms, but changes led to deviation from the authors' original plan to focus wholly on empirical research publications. The analysis mechanism also shifted from a review of research within the context of Ajzen's Theory of Planned Behavior to an exploration of alignment of study findings with the elements of Keller's ARCS Model of Motivation.

CIS methodology also required the authors integrate findings from reviewed publications into their overall question about the efficacy and value of comics in communicating health information. The CIS approach accommodated evolution of the argument as the authors learned more about new (to them) facets of the issue during article analysis. Examining evidence through the lens of the learning-focused ARCS Model of Motivation helped to synthesize the argument and solidify it within the context of education.

Even in selection of the articles for this review, the CIS method's inherent reflexivity enabled the authors to look further outside of the range of literature types and disciplines initially considered relevant, exposing them to concepts that were ultimately invaluable to the argument's development. For instance, the authors found that inclusion of perspective pieces and literary and programmatic criticism provided much needed nuance to their changing conception of comics as a medium for health messaging. Likewise, the framework's flexibility in data extraction enabled inclusion of theoretical works while also accommodating qualitative and quantitative research. 
The CIS approach also facilitated critique of research formats not often favored by more restrictive review methodologies. As multiple studies included in this synthesis focused on historically vulnerable populations (individuals under 18, members of socially disenfranchised or economically disadvantaged groups, etc.) and took place in settings with limited resources, conduct of such studies according to "gold standard" research methods like randomization and "blinding" of participants would have been unethical or unrealistic. The CIS method not only enabled inclusion of these research formats, but also provided a framework where such publications could be considered equally.

Finally, the CIS approach does not require detailed presentation of search and review strategies. This practice was at first challenging for one of the authors who was used to other review methodologies with requirements for recording each search step, as well as the at times arbitrary numbers of results. This difference is especially fortuitous, though, given the continual interpretation and reinterpretation required by the CIS process.

\section{IMPLICATIONS AND CONCLUSION}

At least one component of the ARCS Model was found to be a theme in every evaluated work, although no one component was deemed to be present in every work. Findings from this study suggest that the ARCS Model of Motivation can be used when evaluating health promotional materials, especially when focusing on mediums or methods of implementation. Though used predominantly within the field of education, our findings indicate the ARCS Model likely has utility within health promotion, both in planning and in evaluation.

In addition to support for the ARCS Model's use in evaluating health information and education interventions, our findings indicate that Dixon-Woods' and colleagues' CIS methodology aligns with this purpose as well. Researchers learn and gain new insights throughout the research process, even (or especially) when conducting a synthesis. While other methods of review require maintained focus on the original hypothesis and require much backtracking if researchers' findings open them to different possibilities, CIS encourages critical exploration and embraces an iterative process for perusing the literature and identifying publications for analysis. When investigating a concept such as the engagement offered by comics providing an effective conduit for disseminating communicable disease information, the twists of the topic particularly lend themselves to such a responsive format.

Findings of this synthesis do indicate that comics can be an effective means of providing communicable disease information. Comics are able to capture the attention of their consumers and creators with a mixture of humor, emotion, color (or the lack thereof), and creativity. With proper targeting of both images and text melded with a cogent topic, comics can be quite relevant to their consumers and creators. The image-focused nature of comics enables them to impart information and increase confidence in understanding that information for individuals having varying levels of literacy or prior knowledge of a topic. And comics may inspire enjoyment or a feeling of accomplishment or relief in readers and creators. These factors impact individuals' motivation to learn and act.

While no study or theoretical work analyzed claimed a particular comic intervention was wholly successful or applicable across a range of individuals, comic interventions did appear to impart information in a manner that remains with consumers and, in some cases, helped to change consumers' intention to act. Research also suggests comics created by a target audience for that same audience provide not just relatable materials for comic 
consumers, but lasting impact through learning about a topic (e.g., a particular communicable disease, illustration, etc.) and feeling that one has made a contribution to others. Health educators may want to consider not just existing comics as a means of imparting information, but also comic creation.

Research indicates that comics are likely a useful and effective medium for communicating messages about health promotion, particularly in the often emotionally fraught area of communicable disease. Both traditional comics using cartoon imagery and fotonovelas were useful in creating content that can be relatable to the target audience. Comics hold users' attention and communicate concepts by telling stories with relevant characters, settings, and situations. They can support self efficacy and are accessible, even at different reading levels. They may even inspire feelings of enjoyment and accomplishment in their consumers and creators. These aspects help make comics an engaging medium, and may give them more staying power than text based materials when the content focuses on the human and emotional aspects of communicable diseases.

\section{References}

Aguolu, Obianuju Genevieve. 2018. "Development and Evaluation of a Theory-informed, Culturally Specific Educational Human Papillomavirus (HPV) Vaccine Comic Book for College Students in Northeast OHIO: An Application of the Integrated Behaviour Model (IBM).” Kent State University.

Ajzen, Icek. 1991. "The Theory of Planned Behavior." Organizational Behavior and Human Decision Processes 50 (2): 179-211. doi: https://doi.org/10.1016/0749-5978(91)90020-T.

. 2002. "Perceived Behavioral Control, Self-efficacy, Locus of Control, and the Theory of Planned Behavior." Journal of Applied Social Psychology 32 (4): 665-83. doi: https://doi.org/10.1111/j.15591816.2002.tb00236.x.

Albright, Kendra S., and Karen Gavigan. 2014. "Information Vaccine: Using Graphic Novels as an HIV/ AIDS Prevention Resource for Young Adults.” Journal of Education for Library and Information Science 55 (2): 178-85.

Al-Tawfiq, Jaffar A., and Didier Pittet. 2013. "Improving Hand Hygiene Compliance in Healthcare Settings using Behavior Change Theories: Reflections." Teaching and Learning in Medicine 25 (4): 374-82. doi: https://doi.org/10.1080/10401334.2013.827575.

Bakker, Arnold B. 1999. "Persuasive Communication about AIDS Prevention: Need for Cognition Determines the Impact of Message Format." AIDS Education \& Prevention 11 (2): 150-62.

Bales, Stephen, and Charlie Gee. 2013. "Critical Interpretive Synthesis for Informing Collection Decisions." Collection Building 32 (2): 51-56. doi: https://doi.org/10.1108/01604951311322020.

Bawden, David. 2012. "On the Gaining of Understanding: Syntheses, Themes and Information Analysis." Library \& Information Research 36 (112): 147-62. doi: 10.29173/lirg483.

Beck, Rose Marie. 2006. "Popular Media for HIV/AIDS Prevention? Comparing Two Comics: Kingo and the Sara Communication Initiative." Journal of Modern African Studies 44 (4): 513-41. doi: doi:https://doi.org/10.1017/S0022278X06002072.

Bradshaw, Amanda S., Summer S. Shelton, Easton Wollney, Debbie Treise, and Kendra Auguste. 2021. "Pro-vaxxers Get Out: Anti-vaccination Advocates Influence Undecided First-time, Pregnant, and New Mothers on Facebook." Health Communication 36 (6): 693-702. doi: https://doi.org/10.1080/10410 236.2020.1712037.

Branscum, Paul, and Manoj Sharma. 2009. "Comic Books: An Untapped Medium for Health Promotion." American Journal of Health Studies 24 (4): 430-39.

Carnaghi, Andrea, Mara Cadinu, Luigi Castelli, Jeff Kiesner, and C. Bragantini. 2007. "The Best Way to Tell You to Use a Condom: The Interplay between Message Format and Individuals' Level of Need for Cognition.” AIDS Care 19 (3): 432-40. doi: https://doi.org/10.1080/09540120600582013.

Chan, Alvin, Brandon Brown, Enedina Sepulveda, and Lorena Teran-Clayton. 2015. "Evaluation of Fotonovela to Increase Human Papillomavirus Vaccine Knowledge, Attitudes, and Intentions in a Lowincome Hispanic Community." BMC Research Notes 8 (October): 1-10. doi: https://doi.org/10.1186/ s13104-015-1609-7. 
Clark, Matt and Vincent Coppola. 1985. “AIDS: A Growing 'Pandemic'?” Newsweek 105 (17): 71.

Coughlin, Ellen K. 1990. "Looking for the Messages in Batman and Donald Duck: Researchers Turn to the Comics." Chronicle of Higher Education 37 (1): A5.

Czerwiec, MK. 2018. "Representing AIDS in Comics." AMA Journal of Ethics 20 (2): 199-205. doi: https:// doi.org/10.1001/journalofethics.2018.20.2.mnar1-1802.

Dahl, Ken. 2009. Monsters. Palm Springs, CA: Secret Acres Publishers

De Castro Varela Cruz, Gisele, Mardênia Gomes Ferreira Vasconcelos, Sâmia Jardelle Costa de Freitas Maniva, and Rhanna Emanuela Fontenele Lima de Carvalho. 2019. "Construction and Validation of an Educational Technology on Human Papillomavirus Vaccine for Adolescents." Anna Nery School Journal of Nursing / Escola Anna Nery Revista de Enfermagem 23 (3): 1-7. doi: https://doi.org/10. 1590/2177-9465-EAN-2019-0050.

Depraetere, Joke, Christophe Vandeviver, Tom Vander Beken, and Ines Keygnaert. 2020. “Big Boys Don't Cry: A Critical Interpretive Synthesis of Male Sexual Victimization.” Trauma, Violence \& Abuse 21 (5): 991-1010. doi: https://doi.org/10.1177/1524838018816979.

Diamond, Judy, Julia McQuillan, Amy N Spiegel, Patricia Wonch Hill, Rebecca Smith, John West, and Charles Wood. 2016. "Viruses, Vaccines and the Public." Museums \& Social Issues: A Journal of Reflective Discourse 11 (1): 9-16. doi: https://doi.org/10.1080/15596893.2016.1131099.

Dixon-Woods, Mary, Debbie Cavers, Shona Agarwal, Ellen Annandale, Antony Arthur, Janet Harvey, Ron Hsu, et al. 2006. "Conducting a Critical Interpretive Synthesis of the Literature on Access to Healthcare by Vulnerable Groups.” BMC Medical Research Methodology 6 (35): 1-13. doi: https://doi.org/ 10.1186/1471-2288-6-35.

Fish, Douglas G., Sarah J. Walker, Kumara Singaravelu, Robert Fiore, Linda Klopf, Minda J. Hubbard, Abigail Gallucci, Punkin Clay Stephens, and Lester N. Wright. 2008. "Improving Knowledge, Attitudes, and Testing for Communicable Diseases among New York State Inmates." Journal of Correctional Health Care 14 (4): 290-98. doi: https://doi.org/10.1177/1078345808322614.

Flemming, Kate, and Elizabeth McInnes. 2012. "The Use of Morphine to Treat Cancer Related Pain: A Worked Example of Critical Interpretive Synthesis.” In Synthesizing Qualitative Research, edited by Karin Hannes and Craig Lockwood, 59-82. John Wiley \& Sons, Ltd. doi: https://doi.org/10.1002/ 9781119959847.ch4.

Gavigan, Karen, and Kendra Albright. 2015. "Writing from Behind the Fence: Incarcerated Youths and a Graphic Novel on HIV/AIDS.” Journal of Adolescent \& Adult Literacy 59 (1): 41-50. doi: : https://doi. org/10.1002/jaal.425.

Greenblatt, Jordana. 2019. "The Banality of Anal: Safer Sexual Erotics in the Gay Men's Health Crisis' Safer Sex Comix and Ex Aequo's Alex et La Vie d'après." Journal of Medical Humanities 40 (1): 33-51. doi: https://doi.org/10.1007/s10912-018-9535-z.

Ingrand, Isabelle, Alain Verneau, Christine Silvain, and Michel Beauchant. 2004. "Prevention of Viral Hepatitis C: Assessment of Comic Strip-based Information Campaign Targeting Adolescents." European Journal of Public Health 14 (2): 147-50. doi: https://doi.org/10.1093/eurpub/14.2.147.

Jones, James. 2013. "Cartoons and AIDS: Safer Sex, HIV, and AIDS in Ralf König's Comics." Journal of Homosexuality 60 (8): 1096-1116. doi: https://doi.org/10.1080/00918369.2013.776422.

Katz, Mira, Benjamin Oldach, Jennifer Goodwin, Paul Reiter, Mack Ruffin IV, and Electra Paskett. 2014. "Development and Initial Feedback about a Human Papillomavirus (HPV) Vaccine Comic Book for Adolescents." Journal of Cancer Education 29 (2): 318-24. doi: https://doi.org/10.1007/ s13187-013-0604-8.

Kearns, Ciléin, and Nethmi Kearns. 2020. "The Role of Comics in Public Health Communication during the COVID-19 Pandemic.” Journal of Visual Communication in Medicine July: 1-11. doi: https://doi.org/ 10.1080/17453054.2020.1761248.

Keller, John M. 1987. "Development and Use of the ARCS Model of Instructional Design." Journal of Instructional Development 10 (3): 2-10. doi: https://doi.org/10.1007/BF02905780. 2010. Motivational Design for Learning and Performance: The ARCS Model Approach. New York; London: Springer.

Lee, Taejun (David), Seulki Lee-Geiller, and Byung-Kwan Lee. 2020. "Are Pictures Worth a Thousand Words? The Effect of Information Presentation Type on Citizen Perceptions of Government Websites." Government Information Quarterly 37 (3): 1-12. doi: https://doi.org/10.1016/j.giq.2020.101482.

Markoulakis, Roula, and Bonnie Kirsh. 2013. "Difficulties for University Students with Mental Health Problems: A Critical Interpretive Synthesis." Review of Higher Education: Journal of the Association for the Study of Higher Education 37 (1): 77-100. doi: https://doi.org/10.1353/rhe.2013.0073.

Masson, Mimi, Ibtissem Knouzi, Stephanie Arnott, and Sharon Lapkin. 2021. "A Critical Interpretive Synthesis of Post-Millennial Canadian French as a Second Language Research across Stakeholders and Programs." Canadian Modern Language Review 77 (2): 154-88. 
McCloud, Scott. 1993. Understanding Comics: The Invisible Art. New York, NY: HarperCollins Publishers. 2006. Making Comics: Storytelling Secrets of Comics, Manga and Graphic Novels. New York: Harper.

McFerran, Katrina Skewes. 2016. "Contextualising the Relationship between Music, Emotions and the Well-being of Young People: A Critical Interpretive Synthesis.” Musicae Scientiae 20 (1): 103-21. doi: https://doi.org/10.1177/1029864915626968.

McKibbin, Gemma, Cathy Humphreys, and Bridget Hamilton. 2016. "Prevention-enhancing Interactions: A Critical Interpretive Synthesis of the Evidence about Children who Sexually Abuse Other Children." Health \& Social Care in the Community 24 (6): 657-71. doi: https://doi.org/10.1111/hsc.12260.

McNicol, Sarah. 2017. "The Potential of Educational Comics as a Health Information Medium." Health Information \& Libraries Journal 34 (1): 20-31. doi: https://doi.org/10.1111/hir.12145.

Mheidly, Nour, and Jawad Fares. 2020. "Leveraging Media and Health Communication Strategies to Overcome the COVID-19 Infodemic.” Journal of Public Health Policy 41 (4): 410-20. doi: https://doi.org/ 10.1057/s41271-020-00247-w.

Murray-Román, Jeannine. 2019. "Sexile's Counterpathological Pedagogies at the Intersections of Trans*, Exile, and HIV-prevention Experience." Feminist Formations 31 (2): 155-80.

Muzumdar, Jagannath M., and Nicholas L. Pantaleo. 2017. "Comics as a Medium for Providing Information on Adult Immunizations.” Journal of Health Communication 22 (10): 783-91. doi: https://doi.org/10. 1080/10810730.2017.1355418.

Niederdeppe, Jeff, Deena Kemp, Emma Jesch, Leah Scolere, Amelia Greiner Safi, Norman Porticella, Rosemary Avery, Michael Dorf, Alan Mathios, and Sahara Byrne. 2019. "Using Graphic Warning Labels to Counter Effects of Social Cues and Brand Imagery in Cigarette Advertising." Health Education Research 34 (1): 38-49. doi: https://doi.org/10.1093/her/cyy039.

Norton, Hannah F., Margaret E. Ansell, Ariel Pomputius, Mary E. Edwards, Matthew Daley, and Susan Harnett. 2019. "HIV/AIDS Information Promotion at the Library: Creative Campaigns for Young Adults." Journal of the Medical Library Association 107 (2): 222-31. doi: https://doi.org/10.5195/ jmla.2019.588.

Obare, Francis, Harriet Birungi, Bridget Deacon, and Rob Burnet. 2013. "Effectiveness of Using Comic Books to Communicate HIV and AIDS Messages to In-school Youth: Insights from a Pilot Intervention Study in Nairobi, Kenya." African Population Studies 27 (2): 203-15. doi: 10.11564/27-2-441.

Olson, Ray. 2009. "Monsters.” Booklist 106 (6): 29.

Peeters, Frederik. 2008. Blue Pills: A Positive Love Story. Boston: Houghton Mifflin.

Petersen, Inge, Andy Mason, Arvin Bhana, Carl C. Bell, and Mary McKay. 2006. "Mediating Social Representations using a Cartoon Narrative in the Context of HIV/AIDS: The AmaQhawe Family Project in South Africa." Journal of Health Psychology 11 (2): 197-208. doi: https://doi.org/10.1177/13591 05306061180 .

Phan, Thanh G., Richard Beare, Velandai Srikanth, and Henry Ma. 2021. "Sentiments Expressed in YouTube Public Awareness Campaigns: Stroke.” Internal Medicine Journal 51 (6): 971-74. doi: https:// doi.org/10.1111/imj.15367.

Poirier, Mathieu J. P., Karen A. Grépin, and Michel Grignon. 2020. "Approaches and Alternatives to the Wealth Index to Measure Socioeconomic Status using Survey Data: A Critical Interpretive Synthesis." Social Indicators Research 148 (1): 1-46. doi: https://doi.org/10.1007/s11205-019-02187-9.

Richards, Tessa. 1985. "The Pathology of AIDS." British Medical Journal 291 (6509): 1630-31. doi: https://doi.org/10.1136/bmj.291.6509.1630.

Saji, Sweetha, Sathyaraj Venkatesan, and Brian Callender. 2021. "Comics in the Time of a Pan(Dem)Ic: COVID-19, Graphic Medicine, and Metaphors." Perspectives in Biology and Medicine 64 (1): 136-54. doi: https://doi.org/10.1353/pbm.2021.0010.

Scavone, Paola, Valentina Carrasco, Ana Umpiérrez, María Morel, Daniela Arredondo, and Vanesa Amarelle. 2019. "Microbiology can be Comic.” FEMS Microbiology Letters 366 (14). doi: https://doi. org/10.1093/femsle/fnz171.

Sheer, Vivian C., Dion Tse, Tiffany Chan, and Fuyuan Shen. 2018. "Evaluating the Effectiveness of Four Hong Kong Antismoking Cartoon Posters with Humor and Threat Elements." Chinese Journal of Communication 11 (4): 400-418. doi: https://doi.org/10.1080/17544750.2017.1385497.

Smiley, Nina Feldman. 1992. "Response by High Risk Groups to HIV/AIDS Educational Outreach: A Focus Group Report." Yorktown Heights Putnam and Northern Westchester Counties Board of Cooperative Educational Services NY, and Yorktown Heights Ulster County AIDS Consortium NY.

Spiegel, Amy N., Julia McQuillan, Peter Halpin, Camillia Matuk, and Judy Diamond. 2013. "Engaging Teenagers with Science through Comics.” Research in Science Education 43 (6): 2309-26. doi: https:// doi.org/10.1007/s11165-013-9358-x. 
Stockdale, Janine, Marlene Sinclair, and W. George Kernohan. 2014. "Applying the ARCS Design Model to Breastfeeding Advice by Midwives in Order to Motivate Mothers to Personalise their Experience." Evidence Based Midwifery 12 (1): 4-10.

Toroyan, Tamitza, and Priscilla Reddy. 2006. "Participation of South African Youth in the Design and Development of AIDS Photocomics." International Quarterly of Community Health Education 25 (1/2): 149-63. doi: https://doi.org/10.2190/FG42-PQ5G-5352-2238.

West, Rebecca, and Michael J Silverman. 2021. "Social Skills Instruments for Children with Autism Spectrum Disorder: A Critical Interpretive Synthesis." Journal of Music Therapy 58 (2): 121-54. doi: https://doi.org/10.1093/jmt/thaa017.

Williams, Ian. 2012a. "Comic Reviews: Blue Pills: A Positive Love Story." Graphic Medicine. https://www. graphicmedicine.org/comic-reviews/blue-pills-a-positive-love-story/. . 2012b. "Comic Reviews: Monsters." Graphic Medicine. https://www.graphicmedicine.org/comicreviews/monsters/.

Zhang, Ling, James D. Basham, and Sohyun Yang. 2020. "Understanding the Implementation of Personalized Learning: A Research Synthesis.” Educational Research Review 31 (November): 1-15. doi: https://doi.org/10.1016/j.edurev.2020.100339.

Publisher's Note Springer Nature remains neutral with regard to jurisdictional claims in published maps and institutional affiliations. 